\title{
Emilia Pardo Bazán en la prensa francesa. Revue politique et littéraire. Revue Bleue ${ }^{7}$
}

\author{
Rocío Charques Gámez \\ (Lycée Maurice Ravel, Saint-Jean-de-Luz) \\ (Miembro asociado del Laboratorio Langues, Littératures et Civilisations de l'Arc Atlantique, \\ Université de Pau et des pays de l'Adour) \\ rocio.charquesgamez@univ-pau.fr
}

(recibido xuño/2015, revisado decembro/2015)

RESUMEN: En el presente estudio se analiza la aparición de Emilia Pardo Bazán en la revista francesa Revue politique et littéraire. (Revue Bleue). Se realiza un vaciado de los números de la revista entre 1881 y 1922 con el fin de calibrar la importancia de la escritora en la conocida publicación periódica. Para ello, se ha prestado atención tanto a los textos firmados por la autora como a las traducciones de sus obras. Asimismo se han analizado las referencias a doña Emilia para descubrir la imagen que tienen de ella sus contemporáneos franceses.

PALABRAS CLAVE: Emilia Pardo Bazán, Revue politique et littéraire. (Revue Bleue), prensa del XIX, recepción de la literatura española en Francia, literatura española.

ABSTRACT: This article analyses the presence of Emilia Pardo Bazán in the French review Revue politique et littéraire. (Revue Bleue). We have reviewed all the numbers of the Revue Bleue between 1881 and 1922 in order to show the importance of the writer in this publication. We study Pardo Bazán's texts (also the translations of these ones) and the references to the writer to discover her reception in France.

KEY WORDS: Emilia Pardo Bazán, Revue politique et littéraire. (Revue Bleue), journalism XIX, reception of Spanish literature in France, Spanish literature.

En el presente estudio, pretendemos dar a la luz los resultados de la búsqueda de la presencia de Pardo Bazán en la prensa francesa, concretamente en la Revue politique et littéraire. Daremos cuenta tanto de los textos firmados por la autora como de las traducciones de sus obras. Asimismo nos proponemos encontrar las referencias a la escritora en las páginas de esta publicación periódica y analizar, de este modo, la imagen que tienen sus contemporáneos de ella.

Uno de los títulos imprescindibles en la prensa francesa lo constituye la Revue politique et littéraire, conocida posteriormente como la Revue Bleue. Esta revista es una de las más importantes de la época y una de las que disfrutan de gran tirada en el territorio francés. Comienza su andadura en el año 1863 con la publicación de los cursos de la Sorbona, el Colegio de Francia y otras facultades ${ }^{2}$. Conforme pasa el tiempo amplía su lectorado al tomar un punto de vista más generalista. El vaciado realizado

${ }^{1}$ El presente trabajo se enmarca en el proyecto de investigación I+D subvencionado por el Ministerio de Economía y Competitividad "Ediciones y estudios sobre la obra literaria de Emilia Pardo Bazán" (FFI2013-44462-P).

${ }^{2}$ Los datos sobre la revista los extraemos del trabajo de Christophe Charle, Le siècle de la presse (1830-1939) (pp. 174-175). A Eugène Yung como director de la Revue Bleue le siguen Émile Alglave, Alfred Rambaud y H. Ferrari. 
comprende los números de la Revue Bleue publicados en el periodo que va de 1881 a 1922, esto es, hasta un año después del fallecimiento de la ilustre escritora. Decidimos realizar nuestro análisis siguiendo el orden cronológico de la publicación de los textos, con el fin de llevar a cabo un examen global en el que queden patentes los periodos en los que Pardo Bazán ocupa más espacio en las páginas de esta revista. Nuestro objetivo es dar cuenta de los hallazgos de nuestras pesquisas mediante la somera presentación de estas referencias que servirán de base para estudios posteriores más pormenorizados.

Las primeras alusiones a la autora las hemos localizado en 1885, en los artículos de Léo Quesnel sobre escritores españoles contemporáneos. A propósito de este periodista, cabe revisar el epistolario de Pardo Bazán en el que encontramos una misiva dirigida a José María de Pereda en 1886. En ella se advierte la poca disposición de la autora a reunirse con Quesnel en uno de sus viajes a Francia ${ }^{3}$, lo que muestra el poco interés que le confiere a sus trabajos críticos. Recordemos que por estos años doña Emilia suele pasar unos meses en París, donde estudia en la Biblioteca Nacional y se relaciona con los círculos de intelectuales. Por ejemplo, aprovecha sus estancias para reunirse con Savine, futuro traductor de su Cuestión palpitante 4 . Quesnel la cita en dos ocasiones en dos textos diferentes de 1885 y 1886, y le dedica un estudio monográfico en 1885. En primer lugar, en su estudio sobre la figura de Juan Valera (1885) nombra a Pardo Bazán cuando propone la clasificación de los novelistas españoles en dos bandos. Por un lado sitúa a los idealistas, como Alarcón y Trueba, con Juan Valera a la cabeza; y, por el otro, a los realistas como Pardo Bazán, con Palacio Valdés como autor principal. Entre ambos grupos coloca a Benito Pérez Galdós, cuyo realismo califica de verdadero y bueno. Será a este último escritor al que corone como mejor novelista de España ${ }^{5}$.

Habrá que esperar un par de números más para leer el trabajo en el que Léo Quesnel centra uno de sus artículos en la escritora coruñesa. El 26 de diciembre Quesnel la vuelve a incluir en la nómina de jóvenes escritores que siguen la escuela realista, como Palacio Valdés, pero puntualiza cómo la autora menosprecia los aspectos más crudos y vulgares de la nueva escuela. Además se delinea su similitud con Balzac. Se realiza, a continuación, la retrospectiva de su obra llamando la atención sobre su polémico trabajo La cuestión palpitante (1883), cuyo título se critica por parecerle demasiado ambicioso. Pese a este primer comentario, Quesnel elogia su obra y advierte que Pardo Bazán no solo se contenta con exponer teóricamente sus ideas sobre la novela, sino que también las lleva a la práctica con éxito. De sus novelas destaca dos: Un viaje de novios (1881) y La Tribuna (1883), en las que encuentra magníficamente plasmadas sus ideas. Estas novelas son, como sigue arguyendo, realistas en la forma e idealistas en el fondo. La primera es desgranada en unos cuantos párrafos para mostrar el valor de la misma. A la segunda se refiere más rápidamente y la compara con la primera a la hora de remarcar el carácter católico de los personajes. Léo Quesnel advierte claros contrastes en su obra, pues pese a defender en ella ideas liberales, comparte asimismo las conservadoras defendidas por la Iglesia. Por último, le augura un futuro brillante como escritora, hecho que se adivina por su juventud, su situación social y su extrema erudición. Ha sabido, concluye, elegir lo mejor del nuevo movimiento literario. No obstante, opina que le hace falta distanciarse de sus maestros. Las últimas líneas dejan entrever una censura al realismo y, por ende, a ella, por el hecho de pintar cuadros desmesurados a partir de detalles insignificantes de la realidad, lo que constituye, desde su punto de vista, un alejamiento de la naturaleza, ya que esta lleva aparejada en sí misma la idea de armonía.

\footnotetext{
${ }^{3}$ En ella leemos: "Con quien no tuve lugar de hablar de V. ni de nadie, por la razón plausible de que huí como el fuego de su trato, fue con M ${ }^{\text {me }}$ Léo Quesnel, la que le presentó a V. al público francés bajo el extraño nombre de Don María José de [¿Revada?]” (González Herrán (enero-diciembre 1983): "Emilia Pardo Bazán y José María de Pereda: algunas cartas inéditas”, Boletín de la Biblioteca de Menéndez Pelayo, Santander, LIX). El editor de las cartas, González Herrán, añade una nota en la que explica: "Se refiere al artículo de Léo Quesnel, "Littérature espagnole contemporaine. M. María José de Pereda", Revue Politique et Littéraire, París, 19-IX-1885, n. ${ }^{\circ}$ 12, pp. 372-376. A propósito de este nombre hay una curiosa noticia en carta de J. O. Picón a Galdós, fechada en París el 17 de noviembre de 1888: "No he intentado ver a Léo Guesnél [sic] porque sé que está ausente de París. Y, a propósito, me han asegurado que no es monsieur, si no madame, es decir, que bajo aquel seudónimo se oculta una señora". Los editores de estas cartas comentan en nota: "Ni en los repertorios bibliográficos ni diccionarios de autores franceses figura este seudónimo". (S. de la Nuez y J. Schraibman, Cartas del archivo de Galdós, Taurus, Madrid, 1967, p. 162). (N. del A.)" (ibid., nota 45).

4 "La Pardo Bazán pasó largas temporadas en París en 1884, 1885 y 1886 como demuestra su correspondencia” (Clemessy: Emilia Pardo Bazán como novelista. De la teoría a la práctica, Madrid, Fundación Universitaria Española: 128). Por ejemplo, en carta a Pereda fechada en París el 10 de marzo de 1885 , le va informando de sus trabajos en la Biblioteca Nacional de la capital francesa y sus intenciones de conocer a celebridades del mundo literario francés. También le comunica que ha visitado a Savine, que tiene preparada la traducción de su Cuestión palpitante, con quien ha hablado de él y su obra (González Herrán, 1983, op.cit.).

${ }^{5}$ Léo Quesnel: “Juan Valera”: 475.
} 
En 1886, Léo Quesnel redacta un trabajo sobre el movimiento literario en España en el que hace un balance de las publicaciones históricas recientes, sobre un nuevo drama de Echegaray (Vida alegre y muerte triste) y sobre las novelas, cuentos y poesías publicados en España. Evidentemente incluye el nombre de Emilia Pardo Bazán en el apartado que dedica a los novelistas, pero en ellos no aparecen nuevos comentarios a propósito de su obra. Esta ausencia de crítica viene excusada por el hecho de que las novedades literarias de nuestra autora (El cisne de Vilamorta) y las de José María de Pereda (Sotileza) ya han recibido sendos trabajos monográficos. Por este motivo, repasa muy rápidamente las nuevas novelas que se había dejado en el tintero. Estas son Riverita, de Armando Palacio Valdés; Juan Vulgar, de Jacinto Octavio Picón; y Lo prohibido, de Benito Pérez Galdós. Aparte de estos novelistas, Quesnel puntualiza que existe una enorme lista de jóvenes escritores y que la novela española está floreciendo de una manera extraordinaria.

En 1886 también suscita interés su labor como ensayista, pues no hay que olvidar que este año se publica la traducción de La cuestión palpitante en Francia, acompañada de prólogo del traductor. Albert Savine es el encargado de esta labor y elige cambiar el título por Le Naturalisme. La reseña que Maxime Guacher dedica a esta obra aparece en la sección "Causerie littéraire", en el número del 21 de agosto. El crítico explica la modificación del título en las primeras líneas, donde subraya la impresión de perplejidad que habría causado en el público francés el mantener el original. Anteriormente Quesnel había hecho hincapié también en lo poco acertado del título original, pues el asunto había dejado de ser novedoso y habría provocado la desorientación del lectorado. Por otro lado, Guacher se lamenta de que el traductor haya recortado la parte dogmática y filosófica del ensayo por parecerle árida, y le insta a incluirla en próximas ediciones ${ }^{6}$. Emilia Pardo Bazán lamenta, a su vez, esta supresión y consigue que en las siguientes ediciones se incluya. Aparte de la introducción de estos capítulos en la cuarta edición (1891), las otras novedades que entonces aparecen son el prólogo de Pardo Bazán y dos textos de Zola (una carta dirigida a Savine y la entrevista que le hace Rodrigo Soriano para La Época) ${ }^{7}$. El padre del Naturalismo francés no esconde la admiración que le produce este trabajo realizado por la joven escritora. Asimismo, Gaucher resalta la originalidad de algunas apreciaciones de Pardo Bazán, aunque le reprocha la influencia que sobre ella ejerce la escuela, lo que conduce a doña Emilia a censurar, a veces en exceso, a escritores idealistas como Victor Hugo. El crítico destaca, por tanto, la reflexión teórica llevada a cabo en este libro, en el que no deja de advertir ciertas ideas originales, aunque censura su apego a las teorías naturalistas, sobre todo en el momento de exponer sus ideas a propósito de autores idealistas. Sin embargo, Guacher olvida nombrar las influencias de la tradición literaria española en doña Emilia, lo que le confiere esa originalidad que se destaca en el artículo. Pensamos, sobre todo, en la huella de Cervantes, modelo que Pardo Bazán recordará durante toda su trayectoria desde, por ejemplo, los "Apuntes autobiográficos" que acompañan su novela Los pazos de Ulloa (1886). En ellos rememora sus lecturas infantiles, entre las que se contaban el Quijote, la Biblia y la Ilíada. Por otra parte, el prólogo de Zola incluido posteriormente, en 1891, legitima la seriedad e importancia de la labor crítica de la española en Francia, que entra por la puerta grande a la palestra literaria del otro lado del Pirineo. Sin embargo, no hemos encontrado ninguna mención sobre esta nueva edición en la Revue Bleue, lo que no deja de asombrar por la relevancia del acontecimiento.

Hasta 1891 no hay nuevas referencias a la escritora. En este momento Emilia Pardo Bazán ha comenzado a publicar en Madrid su revista unipersonal Nuevo Teatro Crítico, tarea faraónica que podrá Ilevar adelante durante únicamente tres años, pero que constituye una muestra extraordinaria de su labor poligráfica. La revista tiene una acogida expectante en los medios españoles y la aparición de cada número despierta casi siempre alguna polémica de la que doña Emilia se sirve para promocionar su obra $^{9}$. El número del 16 de mayo de 1891 de la revista francesa introduce una breve nota sobre la aparición de esta publicación. La inclusión de esta nota en la sección dedicada a las noticias del extranjero

\footnotetext{
${ }^{6}$ Savine justifica esta eliminación en el prólogo: "Un tanto árida y escolástica sólo podía interesar a una fracción tan respetable como escasa del público ilustrado" (citado por Faus (2003), Emilia Pardo Bazán. Su época, su vida, su obra, A Coruña, Fundación Pedro Barrié de la Maza, vol. I, nota 10: 226).

${ }^{7}$ Pilar Faus (2003), op.cit., vol. I: 226-227. La edición de 1891 constituye el primer tomo de las obras completas de la autora. Además de los nuevos textos recién citados, se mantienen los prólogos de Clarín (escrito para la segunda edición) y de Savine (realizado para la traducción francesa). González Herrán (1989): "Estudio introductorio" en Emilia Pardo Bazán, La cuestión palpitante, Universidad de Santiago de Compostela, Anthropos: 61.

${ }^{8}$ Nelly Clemessy, op.cit., p. 10.

${ }^{9}$ Sobre la recepción de la revista consúltese nuestro artículo "El Nuevo Teatro Crítico a través de los ojos de sus contemporáneos" (2009).
} 
"Nouvelles de l'Étranger" constituye un gran logro en la tarea publicitaria de Pardo Bazán. Se recuerda, como es previsible, la influencia del Teatro crítico de Feijoo, imprescindible a la hora de trazar las huellas en la formación de la escritora; así como la influencia del Spectator de Addison referencias apuntadas por la misma autora en la presentación de su revista. Tras una breve descripción de las secciones del Nuevo Teatro Crítico, se anota la certidumbre en el éxito de la publicación periódica a partir de la lectura de los cuatro primeros números. Para finalizar se resaltan las dotes de ensayista de Pardo Bazán, así como su curiosidad infinita, su espíritu abierto y la facilidad para abordar con igual calidad cualquier tema que se propone. Junto a esta breve nota se encuentran otras sobre novedades literarias, estrenos, exposiciones artísticas o conferencias, entre otros asuntos. En la misma rúbrica, junto a comentarios sobre Alemania o Inglaterra, alcanzamos a leer dos noticias sobre España: la que acabamos de recoger y otra dedicada a la aparición de la novela La Espuma, de Armando Palacio Valdés. Si bien podemos leer algunas referencias a las novedades literarias españolas, estas siempre serán menos recurrentes que las dedicadas a países como los citados o a Italia, a las que se presta mayor atención en la Revue Bleue.

Un año después, en la primera página del número del 25 de junio, leemos un trabajo de Arvède Barine sobre la polémica novela del jesuita Luis Coloma, Pequeñeces ("Un jésuite romancier. El Padre Luis Coloma"). Esta historiadora y crítica literaria, cuyo verdadero nombre es Louise-Cécile Vincens (18401908) ${ }^{10}$, participa en conocidas revistas de la época como esta o la famosa Revue des Deux Mondes ${ }^{11}$. Su reseña ocupa varias páginas y en ella se menciona a la prestigiosa escritora gallega. De hecho, se la nombra nada más comenzar el texto con el objetivo de contextualizar una breve nota biográfica del escritor. La cita se extrae del libro El Padre Coloma, publicado en Madrid en 1891. Conviene recordar que anteriormente, Pardo Bazán publica su trabajo sobre el novelista y su obra en su Nuevo Teatro Crítico, en abril de 1891, y lo titula "Un jesuita novelista. El padre Luis Coloma" (núm. 4, abril de 1891). Como puede observarse el título de Barine traduce el de la escritora coruñesa en un claro homenaje a ella. Además, las citas que se incluyen en la introducción son las mismas que doña Emilia elige en su estudio. A continuación, comienza la reseña a Pequeñeces, en la que no aparece ninguna otra mención a otros estudios sobre la novela. En cuanto a la acogida del público de la obra del jesuita es de sobra conocido el gran debate que produce en España, pues muchos ven en ella una novela "de clave" que escondía los nombres de conocidas figuras de la alta sociedad española ${ }^{12}$. Numerosas críticas hacen su aparición en la prensa del momento, pero pocos trabajos existen que sean de la amplitud y calidad del realizado por Pardo Bazán. El hecho de que su libro se cite demuestra que se convierte en un texto fundamental a la hora de abordar el análisis de Pequeñeces y, a su vez, pone de relieve la alta concepción que se tiene, en términos generales, en Francia, de la labor crítica de Pardo Bazán. La cita de la misma en las primeras líneas, traducida al francés, es un escaparate ideal para la autora de Los pazos de Ulloa, cuyo nombre es de sobra conocido entre los intelectuales franceses. El estudio de Barine se abre, como hemos visto, con la introducción que se toma de los estudios de Pardo Bazán. Seguidamente, se evoca el contexto en el que transcurre la acción y el argumento de Pequeñeces. Las escenas que se resaltan son aquellas en las que aparecen los hijos de Currita, en concreto, los momentos de mayor dramatismo de la obra. También se menciona la burla que sufre Currita de manos de la amante del marqués de Sabadell. Por último, se hace un balance de la situación de la aristocracia española. Pero se observa que al igual que pueden censurarse los vicios de esta clase, también las otras muestran defectos criticables -hecho apuntado asimismo por doña Emilia. Finalmente se defiende a este estamento y se señala al verdadero culpable de estos vicios en la aristocracia: la institución que la instruye, es decir, la Compañía de Jesús. Para terminar, advierte que se rumorea que Coloma prepara un nuevo libro. En su biblioteca, apunta, se encuentran grandes autores: las obras maestras de la novelística española, la colección de Paul Féval y

\footnotetext{
${ }^{10}$ Wilfert-Portal sostiene que el verdadero nombre de Barine es Thérèse de Solms-Blanc, prestigiosa articulista cuya firma aparece en numerosos periódicos y revistas de la época, y que forma parte del grupo de articulistas cuyos trabajos promocionan la literatura de otros países (Wilfert-Portal (2002/4): "Cosmopolis et l'homme invisible. Les importateurs de littérature étrangère en France, 1885-1914", Le Seuil. Actes de la recherche en sciences sociales, núm. 144: 38). ${ }^{11}$ Isabelle Ernot (2007/1): "L'histoire des femmes et ses premières historiennes (XIXe-début XXe siècle)", Revue d'Histoire des Sciences Humaines, núm. 16: 175. Conocida como la "Sainte-Beuve féminin", comienza sus colaboraciones en prensa en la Revue des Deux Mondes, pero sus inicios literarios datan de 1879. Como se nos explica, "Elle remet périodiquement des articles ou chroniques à la Bibliothèque Universitaire (1876-1885), à la Revue Bleue (1878-1896), à la Revue des Deux Mondes (1879-1899); et, plus épisodiquement, à la Nouvelle Revue (1833-1890); à la Revue de Paris (1895-1905); à Cosmopolis (1897); ainsi que des feuilletons au Journal des Débats. Elle y traite des problèmes sociaux ou philosophiques et marque surtout une curiosité très ouverte aux pays étrangers" (Gabriel Tarde, 2005/2: Cahier 19, Revue d'Histoire des Sciences Humaines, Ed. Sc. Humaines, núm. 13: 179, nota 128).

${ }^{12}$ Enrique Rubio Cremades (2001) hace un resumen de la recepción de la obra en España en su Panorama crítico de la novela realista-naturalista española (Madrid, Editorial Castalia: 569-582).
} 
una selección de obras de Goncourt, Flaubert, Dumas y Zola. Según Bardine, su próxima misión surgirá de estas lecturas profanas, que hacen de él "le missionnaire fin de siècle" (806).

Hasta el momento no se rastrea ningún texto de carácter literario en la revista. Hay que esperar hasta 1896 para ver aparecer la inclusión de uno de los cuentos de la escritora gallega. Su obra cuentística es abundante, en realidad podemos calificarla de descomunal. El profesor González Herrán cuenta más de seiscientos relatos conocidos $^{13}$. El número de relatos publicados hasta 1896 era ya considerable y la calidad de los mismos, incuestionable. Recordemos que a partir del año 1891, Emilia Pardo Bazán cultiva más sistemáticamente este género, como apunta Paredes Núñez en su clásico trabajo sobre el tema ${ }^{14}$. Se nos plantea la pregunta de por qué no se había introducido antes algún relato corto pardobazaniano. De hecho, varios cuentos y cuadros de costumbres ya se habían publicado en La Nouvelle Revue Internationale, entre 1883 y $1887^{15}$. Ana María Freire realiza un balance de las traducciones de la obra de Pardo Bazán de las que tenemos noticias y sitúa la primera traducción de una de sus obras en 1883, en Francia ${ }^{16}$. El primer cuento incluido en la Revue Bleue es "El milagro del hermanuco", editado primeramente en España en La Voz de Guipúzcoa, el 15 de octubre de 1892, y recogido después en el volumen Cuentos nuevos (1894). El 18 de abril de 1896 leemos en la Revue Bleue la traducción realizada por M. A. Chevalier bajo el título "Le miracle du Sacristan. Nouvelle".

Un año después la revista edita otras dos traducciones de cuentos de Pardo Bazán incluidos también en la misma colección. Esta vez los relatos seleccionados son "Piña la Cubaine" y "Sedano". En esta ocasión los títulos van encabezados con el rótulo "Deux contes espagnols" frente al de "Nouvelle" del primero, lo que inmediatamente remite a la procedencia de la autora. De ambas traducciones se encarga G. Art. En cuanto a las versiones originales podemos indicar que "Piña" aparece por primera vez en La Ilustración Artística el 21 de julio de 1890, mientras que "Sedano" lo hace tres años después en El Liberal, el 24 de abril de 1893.

Su conferencia leída en París, "L'Espagne d'hier et celle d'aujourd'hui. La mort d'une légende", va a ocupar un espacio privilegiado en La Revue Bleu. En la época en la que se publica, Freire nos señala que doña Emilia ya colaboraba en La Nouvelle Revue Internationale y en la Revue des Revues ${ }^{17}$. De esta conferencia la crítica actual muestra su interés por la exposición del pensamiento regeneracionista de la autora, que estudian González Herrán ${ }^{18}$ y Sotelo Vázquez ${ }^{19}$. No es la primera vez que expone estas ideas, que asoman en fechas más tempranas como en el cierre de su revista Nuevo Teatro Crítico (1893). Este ideario aparece, igualmente, en su "Discurso inaugural del Ateneo de Valencia", meses después de su conferencia en París (el 29 de diciembre de 1899), o en sus colaboraciones en La llustración Artística, Helios o $A B C^{20}$. En "L'Espagne d'hier et celle d'aujourd'hui", Pardo Bazán intenta borrar el desastroso influjo de la leyenda dorada y la leyenda negra de España, y se une a la minoría intelectual que trata "de reemplazar el ideal legendista por el ideal de la renovación, del trabajo del esfuerzo" (1899: 89).

Emile Berr, en la sección "Petite chronique des lettres", se interesa por esta intervención de Pardo Bazán. En primer lugar, el 1 de abril de 1899 anuncia que nuestra escritora va a dar un discurso el día 18 de este mes sobre "La littérature espagnole", concretamente en la sala Charras de la Société des Conférences. En segundo lugar, en el número del día 15, en la misma sección, habla de la recepción que le espera a Pardo Bazán en París con motivo de su discurso. Por otro lado, en este mismo

\footnotetext{
${ }^{13}$ En concreto, González Herrán cuenta unos seiscientos treinta cuentos ("Once cuentos de Emilia Pardo Bazán recuperados de la revista Caras y Caretas (Buenos Aires, 1909-1916)”, Siglo diecinueve (Literatura hispánica), núm. 16, 2010: 243).

${ }^{14}$ Paredes Núñez (1979): Los cuentos de Emilia Pardo Bazán, Universidad de Granada: 6-7.

${ }^{15}$ Clemessy, op.cit.: 116 .

${ }^{16}$ Freire López (2005): "Las traducciones de la obra de Emilia Pardo Bazán en vida de la escritora", La Tribuna: Cuadernos de Estudios da Casa Museo Emilia Pardo Bazán, 3: 21-38. La traducción más temprana localizada hasta la fecha se publica en Francia en 1883, en La Nouvelle Revue Internationale. El cuento publicado se titula Nieto del Cid. En esta revista se han encontrado otros textos de la misma autora (estudiados por Emilia Pérez Romero, 2010). Indica Ana Freire: "Gracias a las cartas conocemos su relación con Albert Savine, traductor de La cuestión palpitante al francés, o con Charles Aimé Waternau, traductor de La Tribuna a esa misma lengua, al que tiene en alta consideración. Y sólo por testimonios epistolares sabemos que fue Lallié el traductor al francés de La mujer española, publicada en Le Correspondant en 1894, y de Un destripador de antaño, y la buena opinión de Pardo Bazán sobre él” (23). Además, continúa exponiendo, en una carta a Blanca de los Ríos fechada en 1902, la autora le comunica un proyecto editorial en Francia (24).

${ }^{17}$ Freire: op.cit. 27.

${ }^{18}$ González Herrán (1998): “Emilia Pardo Bazán ante el 98 (1896-1905)”, El camino hacia el 98 (Los escritores de la restauración y la crisis de fin de siglo), Madrid, Fundación Duques de Soria, Visor: 139- 153.

${ }^{19}$ Sotelo Vázquez (2000): "Emilia Pardo Bazán ante la crisis del 98: La España de ayer y la de hoy, la muerte de una leyenda", en La crisis española de fin de siglo y la generación del 98. Actas del Simposio internacional, [Noviembre, 1998], A. Vilanova y A. Sotelo (eds.), Barcelona, PPU: 355-368.

${ }^{20}$ Sotelo Vázquez (2008): “Aproximación al pensamiento político de Emilia Pardo Bazán”, Alicante, Biblioteca Virtual Miguel de Cervantes. A partir de la edición de 2005 de Luis F. Díaz Larios [et al.] (eds.), Lectora, Heroína, Autora (La mujer en la literatura española del siglo XIX). III Coloquio de la Sociedad de Literatura Española del Siglo XIX (Barcelona, 23-25 de octubre de 2002), Barcelona, Universitat de Barcelona; PPU: 357-367.
} 
número, Jacques Porcher dedica su sección "Ecrivains espagnols contemporains" a la famosa autora gallega. Es evidente la notoriedad que ha alcanzado la escritora gallega en Francia y la importancia que se le otorga a su intervención en la sala Charras. El retrato resalta las cualidades artísticas de la escritora y, como es habitual entre los críticos de la época, pone de relieve elcarácter varonil de la artista ${ }^{21}$-ejemplos al respecto los encontramos por doquier y no solo a la hora de presentar a Pardo Bazán sino también a otras escritoras como Gertrudis Gómez de Avellaneda, como cuando Bretón de los Herreros afirmó sobre esta última: "Es mucho hombre esta mujer"22. La autora rechaza este tipo de etiquetas pues, para ella, en la literatura "no hay varones ni hembras" ${ }^{23}$. Su alejamiento de un estilo literario "femenino" no es aceptado por el rígido censor social que separa los ámbitos que corresponden a hombres y mujeres ${ }^{24}$.

El texto de la Revue Bleue arranca con el recuerdo de Porcher de la Semana Santa que el autor pasa en Madrid cuatro años antes y la lectura que hace entonces del cuento de Pardo Bazán "La sed de Cristo", que se publica en El Imparcial. Surgen voces que atacan este relato, como en la Correspondencia de España, al que doña Emilia responde desde las páginas de El Heraldo. La rememoración de este episodio se introduce a la hora de esbozar su retrato con la clara intención de mostrar el carácter enérgico y polémico de doña Emilia. De hecho, destaca su humor combativo y energía varonil ${ }^{25}$, junto a su mezcla de misticismo y realismo. No es solo conocida en España, declara Porcher, sino en el extranjero y, a través de este trabajo, desea darla a conocer, aunque sea muy rápidamente, a los lectores de la Revue Bleue. Tras un breve bosquejo de su biografía y obra, pretende anotar el ideario e influencias de la autora coruñesa. Al respecto observa que cuando la corriente naturalista llega a España y contra lo que ocurre con algunos de sus contemporáneos, Pardo Bazán no sigue al pie de la letra los postulados de la nueva escuela. Más que al naturalismo francés, doña Emilia sigue las huellas del realismo español tradicional. En este comentario se aprecia una gran diferencia entre este artículo y el de Quesnel, donde no se nombra la huella de los clásicos españoles en la obra de doña Emilia. Porcher no oculta las fuentes documentales que le han servido para redactar el presente escrito. Así, apoya sus argumentos en la historia literaria del padre Blanco cuando habla de las huellas románticas en novelas como Pascual López. Continúa repasando la obra de la artista y destaca, entre todas sus novelas, Los pazos de Ulloa, aunque censura algo que se encuentra, desde su punto de vista, en otras obras literarias extranjeras, esto es, el exceso de personajes y episodios secundarios que diluyen la acción principal. A la segunda parte de la obra, La madre naturaleza, sigue Una cristiana, en la que advierte un cambio de orientación. La influencia idealista se rastrea en sus obras posteriores, al igual que ocurre con otros autores como Pereda. Cita como ejemplo El saludo de las brujas, donde nota que los personajes están más cerca de los de las tragedias clásicas que de las marionetas que presenta la escuela naturalista. Para finalizar, concluye que a las cualidades manifiestas en sus primeros años, se han ido agregando otras que demuestran que ha alcanzado el momento álgido de su talento ${ }^{26}$. Se cierra su retrato con un broche de oro, pues sitúa a Pardo Bazán entre los mejores escritores españoles de la época.

La Revue Bleue recoge el texto "L'Espagne d'hier et celle d'aujourd'hui. La mort d'une légende", al que se acompaña de varias anotaciones sobre la presencia de Pardo Bazán en París ${ }^{27}$. De este modo, en

\footnotetext{
21 "La galantería, la diferencia sexual en suma, es el principal cliché que los historiadores de la literatura han aplicado de modo insistente a la estimación de las escritoras decimonónicas, cliché con el que, además de encasillarlas, se ha distorsionado a menudo la apreciación sobre su obra" (Ezama Gil (2002): "El canon de escritora decimonónicas en las historias de la literatura", Sociedad de Literatura Española del Siglo XIX, II Coloquio. La elaboración del canon en la literatura española del siglo XIX (Barcelona, 20- 22 de octubre de 1999), Barcelona, Universitat de Barcelona / PPU: 152). Únicamente se aplica el calificativo de "femenina" a Carolina Coronado (ibid.).

${ }^{22} \mathrm{Ibid}$

23 “Carta magna", La Época, 3 de junio de 1884, en Obras completas (1973), Madrid, Aguilar, tomo 3: 657 (citado por Maryellen Bieder (1998): “Emilia Pardo Bazán y la emergencia del discurso feminista”, en Breve historia feminista de la literatura española (en lengua castellana), vol. 5, ed. Iris M. Zavala, Barcelona, Anthropos: 76)

${ }^{24}$ Como puntualiza Maryellen Bieder (1998): "Las literatas eran las mujeres femeninas, las que adoptaron insistentemente una postura normativa al inscribirse en el discurso femenino y en la construcción socio-sexual de la mujer vigentes en su día. Toda mujer literaria que violaba las rígidas definiciones normativas de su sexo corría el peligro de verse a sí misma y a su lenguaje acusados de contaminación por lo masculino. De ahí las caracterizaciones, a veces furibundas, de Pardo Bazán y su obra como viriles, cuando no algo peor" (op. cit., p. 78).

${ }^{25}$ De nuevo se subraya un rasgo no propio de su sexo.

26 “Aux qualités de premier ordre que révélaient ses premiers ouvrages: puissance d'observation, profondeur d'analyse, vérité dans les peintures, richesse et force d'expression, elle est en train d'en ajouter d'autres, s'il est permis de noter comme un progrès un choix plus sévère dans les sujets, un rigueur plus grande dans la composition, un goût plus sobre et plus sûr dans la recherche du détail” (461-462). A la maestría en la observación y el análisis, la riqueza y fuerza de expresión, y en el trazo realista de sus pinturas en sus primeras obras, el crítico advierte la inclusión de nuevas virtudes como un mayor rigor en la composición y en la selección de sus temas, así como mayor sobriedad y tino a la hora de seleccionar los detalles.

${ }_{27}$ Doña Emilia, en la nota "Al lector" que incluye en la publicación en español de su conferencia (1899), habla de los antecedentes de su conferencia. De hecho, no es la primera que realiza en lengua francesa, pues en 1896 la Société Philomatique la invita a Burdeos para disertar sobre La literatura española contemporánea. A continuación, expone cómo le llega la invitación, en octubre de 1898, de parte de la Sociedad de Conferencias de París, para leer una
} 
nota a pie de página se nos indica que se trata del texto de la conferencia leída por Pardo Bazán en la Société des Conférences de París, el 18 de abril de 1899. El discurso se publica ese mismo año en las capitales de España y de Francia. Si consultamos algunas de las reseñas a la conferencia de Pardo Bazán publicadas entre abril y mayo de $1899^{28}$, como las que recogen Journal des Debats, L'Evénement, Correspondance politique, La Fronde, Le Siècle, Le Pain y L'Echo, se observa cómo en todas ellas destacan aspectos positivos de la conferenciante. Sin embargo, algunos indican algún defecto, como Le Siècle, que critica que Pardo Bazán no apunte la catastrófica influencia del catolicismo en su país.

La revista francesa es la elegida por doña Emilia para publicar su célebre conferencia y al director de la misma dirige una misiva que sale en las páginas de la Revue Bleue el 29 de abril de 1899. En ella solicita un espacio para insertar algunas anotaciones que han llegado demasiado tarde para acompañar al texto publicado en el número anterior. Desea la escritora indicar las fuentes de su conferencia ${ }^{29}$. En primer término hace hincapié en la actualidad del tema, del que se discute vivamente en España. Cita, por ejemplo, sus entrevistas con políticos, su asistencia a las sesiones del Senado y la Cámara de diputados desde antes de la guerra. Asimismo, se sirve de la gran cantidad de material que le proporciona la prensa, los debates en las Cámaras de comercio y de la Asamblea de Zaragoza. A continuación, la autora nombra los libros de los que se ha servido para la redacción de su conferencia. Estas obras son Los males de la patria, de Lucas Mallada; Pobre España, de Lapoulide; El problema nacional, de Ricardo Macías Picavea; el Prefacio que Santiago Alba escribe al libro A quoi tient la supériorité des Anglo-Saxons, de Edmond Demolins; Psicología del pueblo español, de Rafael Altamira; Idearium español, de Ángel Ganivet; En torno al casticismo, de Miguel de Unamuno. Otros títulos citados son Hacia otra España, de Ramiro de Maeztu; los estudios políticos que Emilio Castelar publica en varias revistas; Évolution politique et sociale de l'Espagne, de Yves Guyot; y Spain, its greatness and decay, de Martin Hume. Por otra parte, Pardo Bazán advierte cómo ha intentado contestar a todas las cuestiones que se le han enviado hasta la fecha. Por ejemplo, indica que no pertenece a ningún partido político y puntualiza que se adherirá a aquel que defienda los asuntos a los que otorga capital relevancia. Estos son la pedagogía, tema imprescindible en el pensamiento regeneracionista de la autora y del que se ocupa a lo largo de toda su vida ${ }^{30}$; la moral en los asuntos de la administración; la reorganización de la armada, punto espinoso por el que ya recibió aceradas críticas en otra época ${ }^{31}$; y la defensa de la unidad nacional, de la patria. En lo que respecta al nuevo gobierno de Silvela, confiesa su apoyo a algunas iniciativas que se dirigen por este camino y le insta a continuar su labor reformista ${ }^{32}$. Su carta finaliza con un agradecimiento a la gran patria intelectual que constituye París, donde ha sido acogida con gran entusiasmo tanto por escritores y prensa, como por el público en general.

Como observamos, esta es la época en que localizamos con mayor frecuencia el nombre de la insigne escritora. Meses después, en marzo de 1900, la Revue Bleue incluye dos cuentos de amor de Pardo Bazán traducidos por Jacques Porcher, el conocido hispanista que había dedicado un artículo a Pardo Bazán en esta revista el año anterior. La etiqueta elegida para encabezar los títulos de los relatos nos indica ya la procedencia de los mismos, pues ambos se incluyen en el volumen Cuentos de amor (1898). Los textos seleccionados son "Primer amor" ("Premier amour") y "La cabellera de Laura" ("La chevelure de Laure").

Cinco años más tarde, en 1905, se la menciona en dos trabajos diferentes: el que Desdevises du Dézert $^{33}$ redacta sobre clericalismo en España, en la parte que se publica el 28 de enero; y el que Gaultier

conferencia. Ofrece, igualmente, algunos detalles de la bienvenida que recibe en el país vecino y de las reacciones posteriores a su intervención (5-12). Puede consultarse este texto en la página web del Proyecto Filosofía en español (2010 filosofía.org).

${ }^{28}$ Referencias consultadas en la página web del Proyecto Filosofía en español (2010 filosofía.org). Pardo

Bazán reúne estas citas en el apartado "Extractos de la prensa internacional" de su libro La España de ayer y la de hoy (101-107).

${ }^{29}$ En la publicación española agrega otras fuentes como son: La política de capa y espada, de E. Sellés; Las desdichas de la patria, de Vital Fité; ¿Nos regeneramos?, del Marqués de Torre-Hermosa; Historia de España en América, de José de Alcázar; Los desastres y la regeneración de España, de José Rodríguez Martínez; La tragedia de América, de A. Pérez Rioja; y el mensaje y programa de la Cámara Agrícola del Alto Ebro presentado por Joaquín Costa (91-98). Texto incluido en la página web del Proyecto Filosofía en español (2010 filosofía.org).

${ }^{30}$ Ayala Aracil (noviembre 2001): "Emilia Pardo Bazán y la educación femenina", Salina. Revista de Lletres, núm. 15: 183-90. Dedicamos un estudio a la importancia de esta temática en el Nuevo Teatro Crítico: Charques Gámez (2003): "La educación femenina", Los artículos feministas en el Nuevo Teatro Crítico de Emilia Pardo Bazán, Centro de Estudios sobre la Mujer de la Universidad de Alicante, Bancaja, Cuadernos de Trabajos de Investigación 5: 68-87.

${ }^{31}$ La polémica se desata cuando en uno de sus artículos sobre la exposición internacional de París en 1889, critica la desorganización de nuestro ejército (Clemessy: op.cit., 530).

${ }^{32}$ Pilar Faus recuerda la amistad de Pardo Bazán con Francisco Silvela, al que la escritora dedica una crónica que se publica en La Ilustración Artística y Americana, en 1905 (tomo II: 435).

${ }^{33}$ Georges Desdevises du Dézert (1854-1942), catedrático de la Universidad de Clermont-Ferrand, especialista en la historia de la España del siglo XVIII (Niño Rodríguez (1988): Cultura y diplomacia: los hispanistas franceses y España de 1875 a 1931, Madrid, CSIC: 161). 
escribe sobre la crisis de la caridad, en el que nombra uno de los cuentos de la escritora, "Crimen libre", y que aparece en el número del 5 de agosto. Ambas referencias resaltan el prestigio de la autora, conocida en los círculos intelectuales franceses. La cita el primero a propósito de los robos en las iglesias, tema que, como nos explica, doña Emilia ya ha denunciado ${ }^{34}$. Otros célebres escritores nombrados en esta tercera parte del estudio $^{35}$ de Desdevises son Emilio Bobadilla y Benito Pérez Galdós. De Bobadilla se cita Vórtice (Madrid, 1902) y del autor canario se menciona su novela Doña Perfecta, donde se lanza una crítica al fanatismo de provincias. También habla de su obra teatral Electra, cuya representación reciente ha levantado un gran revuelo entre los medios más conservadores. De hecho, recuerda el título de una de las reseñas que resulta revelador en este punto: "El crimen de anoche". Pese al carácter revolucionario que parece tener la obra, Desdevises puntualiza que en Francia no causaría ningún alboroto: “[...] a pu sembler bien anodine aux spectateurs parisiens mais qui a soulevé en Espagne tant de colères [...]" (115). Otras obras que atacan a los representantes de la Iglesia se citan a continuación. Aparece una breve mención a un drama popular del sacerdote catalán Pey Ordeix ${ }^{36}$ y después al libro Les Inquisiteurs d'Espagne (París, 1897), de Fernando Tarrida del Mármol. En 1903, se nos cuenta, Madrazo desea la liberación del yugo eclesiástico que sufre el pueblo en un capítulo de su libro Le peuple espagno/ est-il mort? (1903). Por otro lado, Visions d'Espagne, del escritor argentino Manuel Ugarte, expone los efectos negativos de la Iglesia en el país. Desdevises observa el despertar de la masa, pero su influjo es infructuoso por carecer de cultura y libertad. Únicamente aprecia dos partidos opuestos: el de los conservadores y el de los anarquistas. Los conservadores, aliados con la Iglesia, gobiernan desde la Restauración a través de un bipartidismo. Frente a estos partidos enfrenta a José Canalejas, que trata de fundar un partido progresista, y a los republicanos, para resaltar la poca fortuna que tendrían en España si llegaran a gobernar. Otras fuerzas políticas emergentes son los socialistas y los anarquistas, que tampoco parecen disponer de la energía necesaria para imponerse como elección del pueblo. La reforma eclesiástica que acarrearía el progreso de España parece frenada por el panorama político trazado. Es imprescindible, señala, una reforma del clero español llevada a cabo por el episcopado así como una enseñanza laica para mejorar la educación de los españoles. Aunque en ese momento este cambio esté lejano, augura el nacimiento de hombres abiertos al progreso que sacarán a España de su letargo.

Por su parte, Paul Gaultier dedica un artículo a "La crise de la Charité", publicado en varios números de la Revue Bleue (el 22 de julio y el 5 de agosto de 1905). La cita del cuento "Crimen libre" ${ }^{37}$ de Pardo Bazán viene a ejemplificar la idea del asesinato indirecto cuando explica el derecho de cada hombre a la asistencia, y la falta que supone incumplir este deber hacia el prójimo. Se puede dañar tanto por omisión de socorro como por acción, y en los dos casos existe culpabilidad. La temática del cuento es de rabiosa actualidad en el momento en que se publica, en 1891, pues trata de las nuevas tendencias en materia jurídica, asunto al que nuestra autora dedica varios trabajos ${ }^{38}$. Ruiz-Ocaña cuenta, en $L a$ Ilustración Artística, un centenar de artículos aproximadamente en torno a esta cuestión ${ }^{39}$. De hecho, la sociología del criminal está dando a la luz trabajos reveladores y polémicos ${ }^{40}$ que no dejan indiferentes a los intelectuales de la época, entre ellos, a Pardo Bazán. En 1894, la escritora gallega publica en El Imparcia/sus artículos sobre La nueva cuestión palpitante, donde trata las teorías de Lombroso y Nordau $^{41}$. Por otro lado, esta temática se plasma en su novela de 1891 La piedra angular, que guarda concomitancias con el cuento que Gaultier nombra en su trabajo. La relación entre el cuento citado y su

\footnotetext{
${ }^{34}$ Recordemos sus denuncias expresadas en su conferencia sobre La España de hoy y la de ayer (1889): "La blasfemia es un hábito, el robo sacrílego un caso cotidiano. No hay día en que no sea robada alguna humilde iglesia de aldea" (Madrid: 73-74).

${ }^{35}$ Este artículo se publica en tres números: el 29 de octubre de 1904, el 21 de enero de 1905 y el 28 de enero de ese mismo año.

${ }^{36}$ Se trata del drama Paternidad (1901).

${ }^{37}$ Forma parte de los volúmenes Cuentos escogidos (1891), Arco Iris (1895) y Cuentos sacro-profanos (1899).

${ }^{38}$ Charques Gámez (2011): Emilia Pardo Bazán y su Nuevo Teatro Crítico, Madrid, Fundación Universitaria Española: 78. Dedicamos un epígrafe de uno de los capítulos al tratamiento de este caso en el Nuevo Teatro Crítico (ibíd.: 225-231).

${ }^{39}$ Ruiz-Ocaña Dueñas (2003): La obra periodística de Emilia Pardo Bazán en La Ilustración Artística de Barcelona (1895-1916), UNED, Facultad de Filología: 198.

${ }^{40}$ Litvak, Lily (1990): "La sociología criminal y su influencia en los escritores españoles de fin de siglo”, España 1900. Modernismo, anarquismo y fin de siglo, Barcelona, Anthropos: 129-154.

${ }^{41}$ Ruiz-Ocaña: op.cit., 202.
} 
polémica novela La piedra angular ya ha sido apuntada por José Manuel González Herrán y Darío Villanueva, que subrayan no solo la similitud de escenario sino también de situación ${ }^{42}$.

Un nuevo lapso de varios años, esta vez de cuatro, transcurren hasta la aparición de una nueva referencia a nuestra autora en 1909. Alphonse Daudet la cita en una sección que ocupa varios números y que se dedica a los recuerdos de las tertulias literarias de Goncourt. El 17 de abril en sus "Souvenirs autour d'un groupe littéraire" hace un listado de los artistas reunidos en el conocido "Grenier de Goncourt". Se hace una diferenciación de dos grupos: el de los habituales y más íntimos, como Huysmans o Paul Bourget; y los más jóvenes y menos conocidos, entre los que cuenta a la señora Pardo Bazán. Ningún dato más localizamos en estos interesantes recuerdos aunque puede que se nos haya escapado alguna alusión a nuestra escritora, por otra parte, de sobra conocida en el ámbito literario francés, pese a que Alphonse Daudet considere lo contrario.

El último trabajo literario publicado en la revista es una obra teatral de Pardo Bazán, La suerte, cuya traducción al francés sale en el número del 4 de junio de 1910. "Le sort. Dialogue dramatique" es la traducción y adaptación del texto llevada a cabo por Marie C. de Latour, una de las traductoras habituales en la revista francesa. El diálogo dramático en un acto y dos escenas se estrena en Madrid el 5 de marzo de 1904, en el Teatro de la Princesa, y en mayo de 1905 en A Coruña ${ }^{43}$. Como advierte Monserrat Ribao, aunque la obra no parece ser muy bien acogida por la crítica de su época ${ }^{44}$, la actual comienza a interesarse por ella, como puede comprobarse con las interpretaciones de Francisco Nieva o de Margot Versteeg, que aproximan el texto a obras más modernas como las de Valle-Inclán ${ }^{45}$.

Y las pistas sobre la huella de Pardo Bazán en la Revue Bleue se detienen en el volumen de 1911, donde leemos una reseña de Lucien Maury a la traducción de la novela La madre naturaleza ${ }^{46}$. En su sección "Les lettres: CEuvres et idées" del 1 de julio se ocupa de la condesa de Pardo Bazán ${ }^{47}$ y de Selma Lagerlöf ${ }^{48}$. En la reseña se destaca, sobre todo, la calidad del pincel de nuestra escritora, que sabe plasmar con detalle los rasgos de una fisonomía. Resalta el aspecto visual de sus descripciones y el acopio de personajes de su tierra que guarda en su retina y que presenta magistralmente en su novela. Pese a los aciertos en las pinturas que recoge en su prosa, esta decae en los momentos en que hay que ensamblar las historias. Según Maury, "elle ne sait guère ordonner un récit; la psychologie de ses personnages nous déconcerte dès qu'elle les fait agir; elle est un peintre" (27). Llega a suceder que, a veces, interesan más los personajes secundarios que los principales. La novela se salva, según el articulista, por el realismo externo, por el retrato de Galicia y sus habitantes. Se advierte que doña Emilia militó las filas naturalistas y recuerda la nota al respecto que añade James Fitzmaurice-Kelly en su historia de la literatura española (traducida al francés por Henry D. Davray). No obstante, opina que supo alejarse de los excesos de la escuela. Esto provoca que sus textos sobrevivan cuando a esta escuela sigue otra, pese a que se trate de un libro que salió a la luz veinte años antes.

En suma, contamos muy pocas referencias y apariciones de Pardo Bazán en la famosa Revue Bleue. La primera de ellas data de 1885 y la última de 1911. Únicamente encontramos la traducción de cinco cuentos y una obra teatral, en cuanto a la producción literaria. Con firma de la autora se publican también su discurso sobre "L'Espagne d'hier et celle d'aujourd'hui" y una carta dirigida al director donde menciona las fuentes de su conferencia. Además, se incluyen cuatro trabajos sobre la autora. Se trata, por un lado, de los retratos realizados por Léo Quesnel en 1885, en su serie sobre escritores contemporáneos españoles (crítico poco apreciado por doña Emilia), y el de Jacques Porcher, más interesante, en 1899, momento en que su nombre aparece en los medios franceses con motivo de su discurso en la sala Charras. Por otro lado, nos encontramos con reseñas de dos traducciones de su obra (un ensayo y una novela). La primera cambia el título original (Le Naturalisme, traducida por Savine en

\footnotetext{
${ }^{42}$ Darío Villanueva y José Manuel González Herrán (2003): “Introducción”, Emilia Pardo Bazán, Obras Completas. (Cuentos), edición y prólogo de Darío Villanueva y José Manuel González Herrán, Madrid, Biblioteca Castro. Fundación José Antonio de Castro, VII: XXIV.

${ }^{43}$ Datos extraídos del estudio preliminar que Monserrat Ribao hace al Teatro completo de Pardo Bazán (2010: Madrid, Akal: 24).

${ }^{44}$ Patricia Carballal Miñán (2015) sostiene lo contrario en su tesis, donde apunta que a la luz de las críticas que ha recopilado sobre la obra se demuestra lo contrario. Cita ejemplos no solo de la prensa madrileña, sino también gallega, catalana, andaluza o alicantina, además de la prensa americana (El teatro de Emilia Pardo Bazán. Datos para su historia escénica y para su recepción crítica, Tesis de doctorado UDC, Departamento de Filoloxía Española e Latina, Universidade da Coruña: 246-247).

${ }^{45}$ Ibíd., pp. 25-26.

${ }^{46}$ El traductor de la obra de doña Emilia es J. Demarès de Hill y la editorial, Hachette.

${ }^{47}$ En 1908 Alfonso XIII ha otorgado a nuestra escritora el título real de condesa de Pardo Bazán. Faus: op.cit., II: 234.

${ }^{48}$ Se reseña la novela Le vieux Manoir, traducida del sueco por Marc Hélys y editada por Perrin.
} 
1886), y la segunda es Mère Nature, traducida en 1911 por Demarés de Hill. También descubrimos un anuncio de su revista unipersonal Nuevo Teatro Crítico cuando esta comienza su andadura en 1891. Cabe añadir que el nombre de Emilia Pardo Bazán asoma en varias colaboraciones, con un protagonismo diferente, pues va desde el homenaje más evidente en el trabajo sobre el Padre Coloma realizado por Barine, que copia el título de un estudio de Pardo Bazán pero traducido al francés, hasta la referencia para ejemplificar una aseveración, sin mayor detención en la obra, como sucede cuando se nombra su cuento "Crimen libre" o cuando se recuerdan sus críticas a los robos en los lugares de culto. Nos hemos quedado con ganas de encontrar una mayor presencia de Emilia Pardo Bazán en las páginas de la revista francesa, pero su aparición se ciñe a estas referencias, aunque puede que existan otras que no hayamos rastreado. El grueso de los artículos se sitúa en torno a la aparición de su famosa conferencia en París, momento culmen en que se concentra la mayor parte de ellos. Reina un silencio absoluto sobre otras traducciones y discursos en Francia en las páginas de esta revista que dedica poco espacio a novedades españolas en comparación a otros países como Inglaterra, Alemania o Italia. Sobre todo sorprende notar que no hemos localizado una sola mención al fallecimiento de doña Emilia en la Revue Bleue pese al prestigio y conocimiento de la autora en nuestro país vecino. 


\section{TEXTOS PUBLICADOS EN LA REVUE POLITIQUE ET LITTÉRAIRE}

Léo Quesnel (10 octobre 1885): "Littérature espagnole contemporaine. M. Juan Valera", núm. 15, pp. 474477.

Léo Quesnel (26 décembre 1885): "Littérature espagnole contemporaine. Mme Pardo Bazan", núm. 26, pp. 814-816.

Léo Quesnel (19 juin 1886): "Le mouvement littéraire en Espagne. Publications historiques. Un nouveau drame de M. Echegaray. Romans, contes et poésies", núm. 25, pp. 780-787.

Maxime Gaucher (21 août 1886): "Causerie littéraire", núm. 8, pp. 250-252.

"Nouvelles de l'étranger" (16 mai 1891): núm. 20, pp. 640.

Arvède Barine (25 juin 1892): “Un jésuite romancier. Le Pére Luis Coloma", núm. 26, pp. 801-806.

Emilia Pardo Bazán (18 avril 1896): "Le miracle du Sacristain. Nouvelle", núm.16, pp. 523-525.

Mme E. Pardo Bazán (24 juillet 1897): "Deux contes espagnols. Piña la Cubaine. Sedano": núm. 4: 105-107.

Emilia Pardo Bazán: "L'Espagne d'hier et celle d'aujourd'hui", núm. 16, pp. 492-499.

Jacques Porcher (15 avril 1899): "Écrivains espagnols contemporaines. Madame Pardo Bazán", núm. 15, pp. 459-462.

Emile Berr (1 avril 1899): "Petite chronique des lettres", núm. 13, pp. 416.

Emile Berr (15 avril 1899): "Petite chronique des lettres", núm. 15, pp. 480.

Emilia Pardo Bazán (29 avril 1899): "Correspondance", núm. 17, pp. 544.

Emilia Pardo Bazán (31 mars 1900): "Deux contes d'amour. Premier amour. La chevelure de Laure", núm. 13, pp. 394-399.

Desdevises du Dézert (28 janvier 1905): "Le cléricalisme en Espagne”, núm. 4, pp. 114-117.

Paul Gaultier (5 août 1905): "La crise de la charité", núm. 6, pp. 187-191. Emilia Pardo Bazán (4 juin 1910): "Le sort. Dialogue dramatique", núm. 23, pp. 717-719.

Lucien Maury (1 juillet 1911): "Les lettres: Oeuvres et idées. Deux romancières", nº1, pp. 26-29.

Mme Alphonse Daudet (17 avril 1909): "Souvenirs autour d'un groupe littéraire", nº16, pp. 484-488. 


\section{BIBLIOGRAFÍA}

Ayala Aracil, María de los Ángeles (noviembre 2001): "Emilia Pardo Bazán y la educación femenina", Salina. Revista de Lletres, núm. 15, pp. 183- 90.

Bieder, Maryelle (1998): "Emilia Pardo Bazán y la emergencia del discurso feminista", en Breve historia feminista de la literatura española (en lengua castellana), vol. 5, ed. Iris M. Zavala, Barcelona, Anthropos, pp. 75-110

Carballal Miñán, Patricia (2015): El teatro de Emilia Pardo Bazán. Datos para su historia escénica y para su recepción crítica, Tesis de doctorado UDC, Departamento de Filoloxía Española e Latina, Universidade da Coruña.

Charle, Christophe (2004): Le siècle de la presse (1830-1939), Paris, Éd. du Seuil.

Charques Gámez, Rocío (2003): "La educación femenina", Los artículos feministas en el Nuevo Teatro Crítico de Emilia Pardo Bazán, Centro de Estudios sobre la Mujer de la Universidad de Alicante, Bancaja, Cuadernos de Trabajos de Investigación 5, pp. 68-87.

(2009): "El Nuevo Teatro Crítico a través de los ojos de sus contemporáneos", edición de José Manuel González Herrán, Cristina Patiño Eirín y Ermitas Penas Varela, La literatura de Emilia Pardo Bazán, Real Academia Galega, pp. 245-52.

(2011): Emilia Pardo Bazán y su Nuevo Teatro Crítico, Madrid, Fundación Universitaria Española.

Clemessy, Nelly (1981): Emilia Pardo Bazán como novelista. De la teoría a la práctica, Madrid, Fundación Universitaria Española.

Ernot, Isabelle (2007/1): "L'histoire des femmes et ses premières historiennes (XIXe-début XXe siècle)", Revue d'Histoire des Sciences Humaines, núm. 16, pp. 165-194.

Ezama Gil, Ángeles (2002): "El canon de escritora decimonónicas en las historias de la literatura", Sociedad de Literatura Española del Siglo XIX, // Coloquio. La elaboración del canon en la literatura española del siglo XIX (Barcelona, 20-22 de octubre de 1999), Barcelona, Universitat de Barcelona / PPU, pp. 149-160.

Faus, Pilar (2003): Emilia Pardo Bazán. Su época, su vida, su obra, La Coruña, Fundación Pedro Barrié de la Maza, dos volúmenes.

Freire López, Ana María (2005): "Las traducciones de la obra de Emilia Pardo Bazán en vida de la escritora", La Tribuna: Cuadernos de Estudios da Casa Museo Emilia Pardo Bazán, 3, pp. 21-38.

González Herrán, José Manuel (enero-diciembre 1983): “Emilia Pardo Bazán y José María de Pereda: algunas cartas inéditas", Boletín de la Biblioteca de Menéndez Pelayo, Santander, LIX, pp. 259-87.

(1989): "Estudio introductorio" en Emilia Pardo Bazán, La cuestión palpitante, edición de José Manuel González Herrán, Barcelona. Santiago de Compostela, Anthropos, pp. 7-103.

(1998): “Emilia Pardo Bazán ante el 98 (1896-1905)", El camino hacia el 98 (Los escritores de la restauración y la crisis de fin de siglo), Madrid, Fundación Duques de Soria, pp. 139-153.

(2010): “Once cuentos de Emilia Pardo Bazán recuperados de la revista Caras y Caretas (Buenos Aires, 1909-1916)", Siglodiecinueve(Literatura hispánica), núm. 16, pp. 243-90.

Litvack, Lily (1990): "La sociología criminal y su influencia en los escritores españoles de fin de siglo", España 1900. Modernismo, anarquismo y fin de siglo, Barcelona, Anthropos, pp. 129-154.

Niño Rodríguez, Antonio (1988): Cultura y diplomacia: los hispanistas franceses y España de 1875 a 1931, Madrid, CSIC.

Pardo Bazán, Emilia (abril 1891): "Un jesuita novelista. El padre Luis Coloma", Nuevo Teatro Crítico, Madrid, La España Editorial, núm. 4, pp. 31-72.

(1899): La España de ayer y la de hoy, A. Avrial, Madrid. Transcripción del texto contenido en

este opúsculo de 107 páginas <Proyecto Filosofía en español. Url: http://www.filosofia.org/aut/001/1899epb.htm>

Paredes Núñez, Juan (1979): Los cuentos de Emilia Pardo Bazán, Universidad de Granada.

Pérez Romero, Emilia (2010): "L’Espagne dans les articles d’Emilia Pardo Bazán pour la Nouvelle Revue Internationale", La culture de l'autre: I'enseignement des langues à l'Université. Actes 2010. La Clé des Langues, 
Lyon, ENS LYON/ DGESCO. <Url: http://cle.ens-lyon.fr/espagnol/l-espagne-dans-les- articles-d-emilia-pardobaza-769-n-pour-la-nouvelle-revue-internationale- 87341.kjsp>

Ribao, Monserrat (2010): "Estudio preliminar", Teatro completo, Emilia Pardo Bazán, Madrid, Akal, pp. 7-58.

Rubio Cremades, Enrique (2001): Panorama crítico de la novela realista-naturalista española, Madrid, Editorial Castalia.

Ruiz-Ocaña Dueñas, Eduardo (2003): La obra periodística de Emilia Pardo Bazán en La Ilustración Artística de Barcelona (1895-1916), UNED, Facultad de Filología.

Sotelo Vázquez, Marisa (2000): "Emilia Pardo Bazán ante la crisis del 98: La España de ayer y la de hoy, la muerte de una leyenda", en La crisis española de fin de siglo yla generación del 98. Actas del Simposio internacional, [Noviembre, 1998], A. Vilanova y A. Sotelo (eds.), Barcelona, PPU, pp. 355-368.

(2008): "Aproximación al pensamiento político de Emilia Pardo Bazán", Alicante, Biblioteca Virtual Miguel de Cervantes. A partir de la edición de 2005 de Luis F. Díaz Larios [et al.] (eds.), Lectora, Heroína, Autora (La mujer en la literatura española del siglo XIX). III Coloquio de la Sociedad de Literatura Española del Siglo XIX (Barcelona, 23-25 de octubre de 2002), Barcelona, Universitat de Barcelona; PPU, pp. 357-367.

Tarde, Gabriel (2005/2): Cahier 19, Revue d'Histoire des Sciences Humaines, Ed. Sc. Humaines, núm. 13, pp. 141-182.

Villanueva, Darío y José Manuel González Herrán (2003): “Introducción”, Emilia Pardo Bazán, Obras Completas. (Cuentos), edición y prólogo de Darío Villanueva y José Manuel González Herrán, Madrid, Biblioteca Castro. Fundación José Antonio de Castro, VII: IX-XXXIII.

Wilfert-Portal, Blaise (2002/4): "Cosmopolis et I'homme invisible. Les importateurs de littérature étrangère en France, 1885-1914", Le Seuil. Actes de la recherche en sciences sociales, núm. 144, pp. 33-46. 